\title{
Aggressive Posterior Retinopathy of Prematurity (APROP): LASER as the Primary Modality of Treatment
}

\section{Shilpi H Narnaware'1, ICO, FAICO; Prashant K Bawankule², FMRF, Hon FAICO; Dhananjay Raje ${ }^{3}$, CStat, PhD}

${ }^{1}$ Consultant Vitreo-retina \& ROP Specialist, Sarakshi Netralaya, Rajiv Nagar, Wardha Road, Nagpur 440025, Maharashtra, India ${ }^{2}$ Vitreo-Retinal Surgeon, Rajiv Nagar, Wardha Road, Nagpur 440025 Maharashtra, India

${ }^{3}$ Head, Data Analysis Group, MDS Bio-analytics Pvt. Ltd., Shankar Nagar, Nagpur 440010, Maharashtra, India

ORCID:

Shilpi H. Narnaware: https://orcid.org/0000-0003-3170-955X

\section{Abstract}

Purpose: To study the success rate of LASER as a primary modality of treatment in aggressive posterior retinopathy of prematurity (APROP) cases.

Methods: This is a prospective case series of 56 eyes of 28 preterm babies (males $=21$ ) with APROP who underwent laser therapy. Babies were divided into groups on the basis of gestational age (GA), birth weight (BW), and postmenstrual age (PMA) at which treatment was performed. GA (in weeks): $<28(n=7), 28-30(n=11),>30(n=10)$. BW (in grams): $<1000$ ( $n=8), 1000-1200(n=10),>1200(n=10)$. PMA (in weeks): $<32(n=6), 32-34(n=18)$, $>34(n=4)$. Success was calculated as complete regression of disease without need for any other modality of treatment such as anti-vascular endothelial growth factor (anti-VEGF) or pars plana vitrectomy.

Results: The overall success rate was $94.64 \%$ (53/56). Two babies who needed additional modality of treatment were $<28$ weeks of GA (one eye) and 28-30 weeks (two eyes). One baby (one eye) was $<1000 \mathrm{gm}$ and the other (two eyes) was $>1200 \mathrm{gm}$, while PMA at which additional treatment was needed was 30 weeks in one baby (one eye) and 33 weeks in the other (two eyes).

Conclusion: In this era of anti-VEGF treatment, even in cases of APROP, LASER should still be considered as a primary modality of treatment, as it is a one-time treatment without the concern of systemic side effects and recurrent/persistent avascular zones.

Keywords: Aggressive Posterior Retinopathy of Prematurity; LASERS; Success Rate

J Ophthalmic Vis Res 2021; 16 (3): 400-407

\section{INTRODUCTION}

Aggressive posterior retinopathy of prematurity (APROP) is a distinct variant of retinopathy of

Correspondence to:

Shilpi H. Narnaware, MBBS, D.O., Fellowship in VitreoRetina, ICO (Retina). Sarakshi Netralaya, Plot No. 19, Rajiv Nagar, Wardha Road, Nagpur 440025, Maharashtra, India.

E-mail: shilpi.narnaware@gmail.com

Received: 08-03-2020 Accepted: 15-06-2019

\section{Access this article online}

Website: https://knepublishing.com/index.php/JOVR prematurity (ROP) which does not respect various stages and can rapidly lead to blindness if untreated. According to various studies, ${ }^{[1-4]}$ the overall incidence of ROP varies from $38 \%$ to $51.9 \%$ in the Indian subcontinent. Nearly $26.4 \%$ of babies needed treatment for one of the stages of ROP ${ }^{[4]}$ almost half of them had APROP. ${ }^{[4]}$

This is an open access journal, and articles are distributed under the terms of the Creative Commons Attribution-NonCommercial-ShareAlike 4.0 License, which allows others to remix, tweak, and build upon the work non-commercially, as long as appropriate credit is given and the new creations are licensed under the identical terms.

How to cite this article: Narnaware SH, Bawankule PK, Raje D. Aggressive Posterior Retinopathy of Prematurity (APROP): LASER as the Primary Modality of Treatment. J Ophthalmic Vis Res 2021;16:400-407. 
Many previous studies have shown more unfavorable outcomes in APROP (ranging from $15 \%$ to $29 \%$ ) undergoing laser treatment compared with anti-vascular endothelial growth factor (antiVEGF) injection. ${ }^{[5-8]}$ Because of these unfavorable outcomes, alternatives in treatment of APROP were explored over the last few years. The first prospective, controlled, randomized trial was performed by Mintz-Hittner ${ }^{[9]}$ showing significantly lower recurrence rate following intravitreal bevacizumab (IVB), compared to laser photocoagulation, especially in Zone 1 ROP. After that, several studies reported the success rate of around 85\% in Zone 1 and APROP with IVB monotherapy. ${ }^{[10,11]}$ However, none of these studies talked about the systemic side effects, recurrence rates, and need of laser treatment after IVB in their studies.

In this study, we prospectively studied the structural success rate in cases of APROP after laser treatment and assessed various parameters like systemic side effects, complications, follow-up period and retreatment.

\section{METHODS}

A prospective case series including 56 eyes of 28 infants with APROP who were treated with laser photocoagulation between January 2015 and June 2018 and were followed-up for 12 months. Various parameters including birth weight (BW), gestational age, postmenstrual age (PMA), neonatal illness risk factors, and oxygenation were studied.

Diagnosis of APROP was made in accordance with the International Classification of $\mathrm{ROP}^{[12]}$ and documented by retinal drawings and/or NEORET images [Figure 1A]. APROP was diagnosed according to the International Classification, ${ }^{[12]}$ which is described as follows: "The characteristic features of this type of ROP are its posterior location, prominence of plus disease, and the ill-defined nature of the retinopathy."

All infants received confluent laser spots (less than half burn width apart) to cover the full avascular retina with green laser (532 nm) delivered through the indirect ophthalmoscopic system under topical anesthesia in the NICU setting. Mean number of laser spots were 4200 t/- 600 per eye. Completion of laser was confirmed by a second observer or NEORET photography. PMA at treatment, number of laser sittings, and outcome were noted. Babies were followed-up for up to 12 months and the outcome was labeled as unfavorable if any of the following three situations/conditions were seen: (1) retinal detachment (stage 4a/4b/5), (2) falciform fold involving the macula, and (3) disc/macular dragging.

\section{Follow-up and Retreatment}

The follow-up examination after laser treatment with NEORET photography [Figure 1B] were performed weekly for one month, biweekly for two months, and then every three months for nine months. In cases where avascular non-covered areas were seen, fill-in laser was applied to the skipped areas usually one or two weeks after the initial laser session. Mean number of laser spots during retreatment was $400 /+-100$ per eye.

\section{Statistical Methods}

The description for various risk factors such as gestational age, BW, PMA, and regression week were obtained in terms of mean and standard deviation. The unfavorable event was treated as dependent variable and the effect of different risk factors was studied on outcome through univariate logistic regression. The odds ratio associated with each factor were obtained and interpreted. The number of babies with different complications were obtained and the association of outcome with the number of complications was represented through a stacked bar chart. Further, the scatter plots showing association of BW with gestational age and PMA were obtained highlighting the unfavorable events. All analyses were performed using SPSS version 20.0 (IBM Corp, USA) and the graphical representations were obtained using Microsoft Excel. A p-value of less than 5\% was considered as statistically significant.

\section{RESULTS}

Fifty-six eyes of 28 preterm babies (21 male and 7 female) with APROP who underwent laser treatment were studied. The mean gestational age of mothers was 28.5 (SD: 2.01) weeks and the mean BW of babies was 1,128 (SD: 310) gr. The mean PMA of mothers was 32.7 (SD: 1.63) weeks. The undesired event occurred in three eyes out 


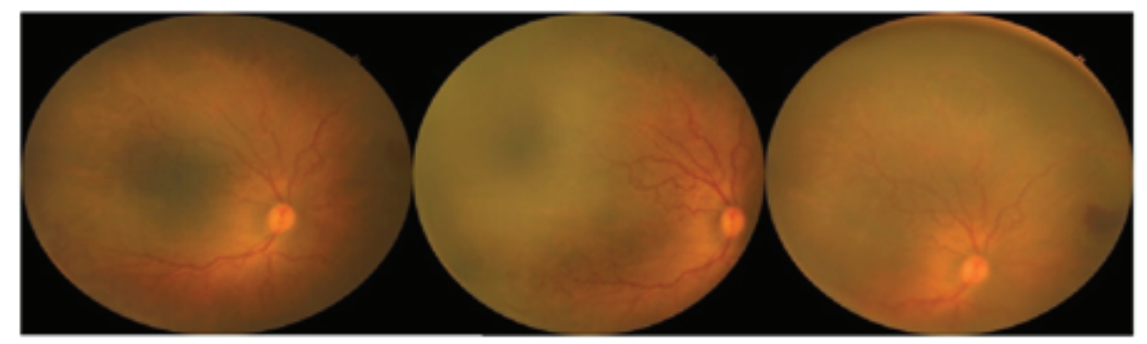

A

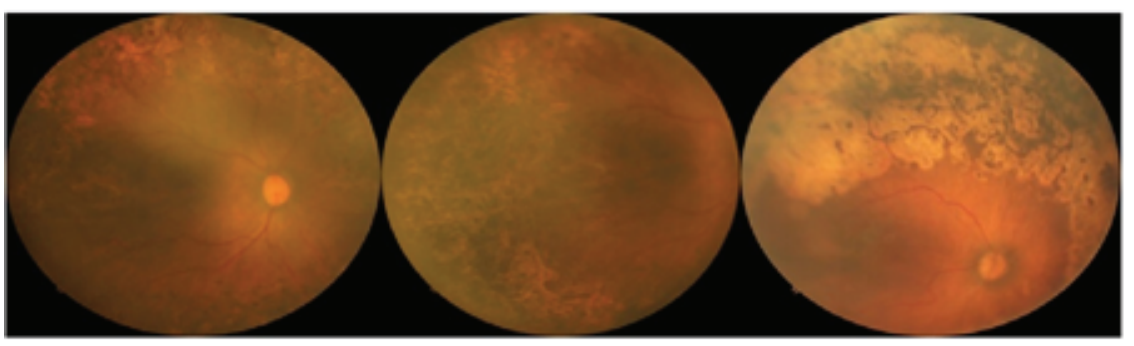

B

Figure 1. Fundus photos of eyes pre- (A) and post-laser (B).

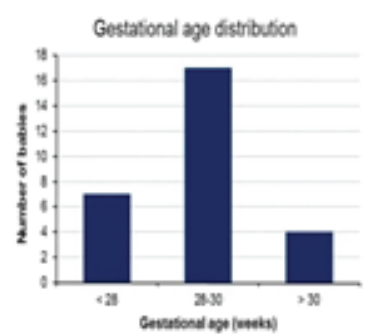

A

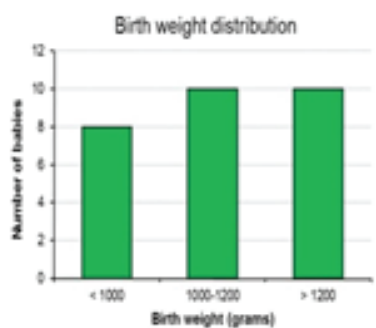

B

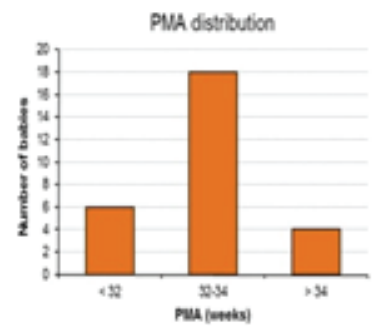

C

Figure 2. Column chart showing number of babies according to gestational age (A), birth weight (B), and PMA (C).

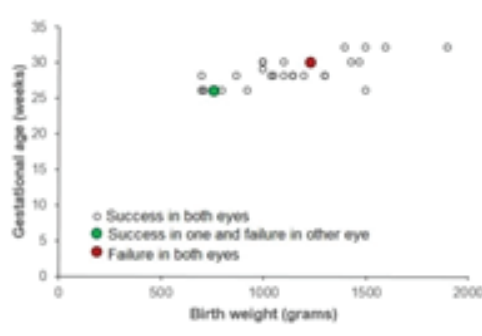

A

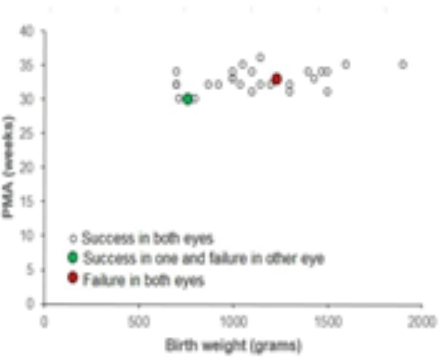

B

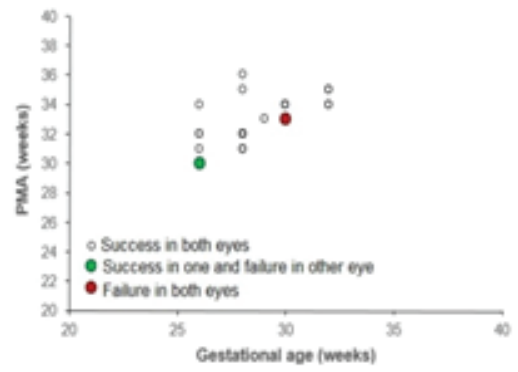

C

Figure 3. Scatter plot showing success corresponding to birth weight and gestational age (A), birth weight and PMA (B), and gestational age and PMA (C). 


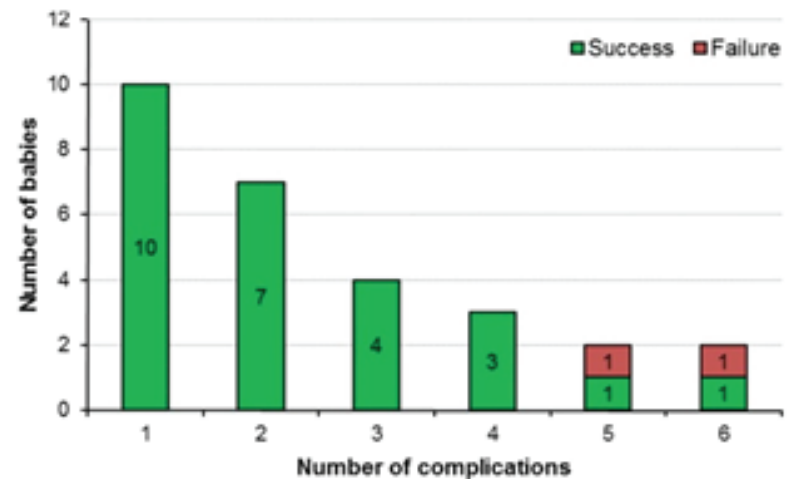

Figure 4. Stacked bar chart showing number of babies according to number of risk factors.

of 56 resulting in the overall success rate of $94.64 \%$. The risk associated with various factors was studied though logistic regression and showed the following results.

\section{Gestational age}

The odds associated with gestational age was 1.036 (95\% Cl: 0.577-1.858) indicating hardly any effect of gestational age on the success rate, and the effect was not statistically significant $(P=$ 0.906).

\section{BW}

The odds associated with BW was 0.999 (95\% Cl: 0.995-1.003) indicating hardly any effect of BW on the success rate, and moreover the effect was statistically insignificant $(P=0.750)$

\section{PMA}

The odds associated with PMA was 0.735 (95\% Cl: 0.339-1.591) indicating that the increase in PMA reduced the risk of failure; however, the effect was not statistically significant $(P=0.434)$.

\section{Regression week}

It was defined as the interval between application of laser to complete regression of the disease. The odds associated with regression week was 3.814 (95\% Cl: 0.446-32.641) indicating that any increase in the regression week increased the risk of failure, although the effect was found to be statistically insignificant $(P=0.222)$.
Figures $2 \mathrm{~A}-2 \mathrm{C}$ show the distribution of babies according to gestational age, BW, and PMA. With regard to gestational age, the majority, that is, $17(60.7 \%)$ babies were born in 28-30 weeks of gestational age. There were 20 (71.42\%) babies with BW >1000 gm; and there were 18 (64.29\%) babies with mothers having PMA between 32 and 34 weeks.

Figures 3A-3C, show the scatter plots demonstrating the structural success rates corresponding to the interplay between GA, PMA, and BW.

Supplementary laser treatment was needed in 20 eyes, one to two weeks after the initial laser treatment. The mean regression period was 4.68 weeks (SD: 0.71) in babies with $\mathrm{BW}>1000 \mathrm{gm}$ and 6.36 weeks (SD: 0.92) when the BW was $<1000 \mathrm{gm}$. No relation was seen with GA or PMA.

Out of the 56 eyes, 53 eyes had complete regression while of the other three eyes, two progressed to stage 5 and one progressed to stage 4A despite laser. One baby (two eyes), which progressed to stage 5 was 30 weeks of GA and $>1200 \mathrm{gm}$ BW, while the other baby (one eye) that progressed to stage 4A was $<28$ weeks and $<1000$ gm BW.

Table 1 illustrates the distribution of various risk factors/systemic illness in babies. Supplemental unmonitored oxygen was the common factor among all the babies. In Figure 4 , the stacked bar chart representation reveals that as the number of risk factors increases, the likelihood of failure increases. Failures were observed in cases with five or more complications. 
Table 1. Number of babies with different complications

\begin{tabular}{lcc}
\hline Complication & Number & $\%$ \\
\hline Oxygen administration & 28 & 100 \\
Neonatal jaundice & 11 & 39.28 \\
Sepsis & 10 & 35.71 \\
RDS & 11 & 39.28 \\
Hypothermia & 5 & 17.86 \\
Shock & 3 & 10.71 \\
Blood transfusion & 2 & 7.14 \\
\hline
\end{tabular}

\section{DISCUSSION}

In the present study, 53 out of 56 eyes had complete regression, while out of 3 eyes without complete regression, 2 progressed to stage 5 and 1 progressed to stage $4 \mathrm{~A}$. Mean regression period was 4.68 weeks in babies with $\mathrm{BW}>1000 \mathrm{gm}$ and 6.36 weeks when BW was $<1000 \mathrm{gm}$. According to previous studies, mean GA and BW of infants with APROP were significantly lower than those with non-APROP ${ }^{[13,14]}$ A study by Tong et al ${ }^{[15]}$ showed that older PMA and low neutrophil count were associated as risk factors for retinal detachment in APROP. Also, low BW was significantly associated with recurrence in APROP. Many studies have shown the beneficial effect of IVB over laser in terms of structural outcome specially in cases of APROP. The studies by Drenser et $\mathrm{al}^{[7]}$ and Pandya et $\mathrm{al}^{[8]}$ reported that $8 / 44$ eyes and $3 / 6$ eyes, respectively, progressed to retinal detachment in spite of laser treatment. While $17 \%$ of eyes landed into retinal detachment in the study by Sanghi et al, ${ }^{[6]}$ in our study only $5.36 \%$ of babies progressed to Stage 4/5 after laser treatment which is much less than the rates reported by previous studies.

Gunay et al from Turkey reported 0/25 and $2 / 11$ eyes progressing to retinal detachment in IVB group versus laser group ${ }^{[10]}$ while Nicoara et al reported the success rate of $94 \%$ in IVB group compared to $83 \%$ in laser group in the Romanian population. ${ }^{[11]}$

Recently, many studies have reported reactivation of disease with IVB. In a study by Lorenz et $\mathrm{al}^{[16]}$ only $25 \%$ of eyes with APROP showed regression with lower doses of bevacizumab, that is, $0.312 \mathrm{mg}$. In a recent study, Mintz-Hittner showed reactivation in $100 \%$ of eyes with APROP ${ }^{[17]}$ while Blair et al found $41 \%$ reactivation in APROP eyes in their study. ${ }^{[18]}$ This difference in reactivation may be because of higher levels of VEGFs in APROP eyes. Because of late reactivation up to 69 weeks+ after anti-VEGF treatment, longer-term follow-up is required. ${ }^{[19]}$ The babies in our study were followed-up for up to one year of age to look for recurrence. The average number of visits in our study was 7-10 in a year. In study by Simona et $a l,{ }^{[20]}$ babies receiving laser needed an average follow-up of up to 60 weeks with an average of 8-9 visits which is much less compared to a follow-up of up to 80 weeks and an average of 16-18 visits in the group receiving anti-VEGF treatment. Persistent avascular zones after IVB have been reported in up to $91.7 \%$ of cases by Leopore et al, ${ }^{[21]}$, while $100 \%$ of eyes needed additional treatment with laser because of persistent avascular zones which were confirmed on fluorescein angiography in a study by Michael Blair et al. ${ }^{[18]}$ In the present study, 53 eyes after regression did not need any additional form of treatment in the form of anti-VEGF injection or surgery. Another concern is of the "crunch phenomenon" with IVB which causes fibrovascular contraction and tractional retinal detachment following intravitreal anti-VEGF. ${ }^{[22]}$ Systemic safety is another concern. Intravitreal injection causes breakdown of blood retinal barrier, ${ }^{[23]}$ hence, anti-VEGF is found in systemic circulation after intravitreal injection causing serum VEGF levels to decrease. ${ }^{[23,24]}$ Various studies demonstrated that serum VEGF plasma levels may be lowered up to two to seven weeks after IVB. ${ }^{[25-27]}$ These decreased VEGF levels may cause adverse effects on VEGF-dependent developments such as the development of brain, lungs, kidney, and normal neural retinal development. ${ }^{[28]}$ Few studies have 
shown delay in growth and pulmonary dysplasia in bevacizumab-treated babies. ${ }^{[29]}$ A study by Morin et al reported that severe neurodevelopmental disabilities (Bayley scores $<70$ ) was seen 3.1 times higher with bevacizumab compared to laser. ${ }^{[30]}$ Most recently, hypotension-related reports ${ }^{[31]}$ and histopathologically proven new or reactivation of bronchopulmonary dysplasia have been noted after anti-VEGF administration. ${ }^{[32]}$

Few studies were designed to study specific abnormalities related to anti-VEGF treatment and detected no systemic complications. ${ }^{29,33,34]}$ These negative results may be due to the fact that infants with ROP present more often with developmental disorders compared to other infants, causing difficulty in assessing systemic side effects of anti-VEGF treatment. ${ }^{[35]}$ None of the babies showed any signs of systemic complications in our case series. Laser therapy in ROP is associated with restricted visual field ${ }^{[36-39]}$ and refractive error. ${ }^{[40,41]}$ It is postulated that increased laser ablation spots might induce more severe myopia and for every 100 laser spots myopia increases by $-0.14 \pm 0.05 \mathrm{D} .^{[42]}$ Prevalence of high myopia reported in literature varies from $8 \%{ }^{[43]}$ to $35 \% .{ }^{[44]}$ Various studies have shown more chance of refractive anisometropia ${ }^{[43]}$ and myopia $^{[40,41]}$ in babies receiving laser therapy compared to IVB. However, the study by Kua et $\mathrm{al}^{[42]}$ and Isaac et $\mathrm{al}^{[44]}$ reported no statistically significant difference in refractive error between IVB and laser groups. Because of conflicting results, one meta-analysis ${ }^{[45]}$ has concluded the need to investigate the long-term effects of IVB therapy on refractive error development. Although, myopia ${ }^{[40,41]}$ and field restriction ${ }^{[36-39]}$ are the main adverse events associated with laser therapy, in our study, we only evaluated the structural success and the refractive status was not evaluated.

This study had a few limitations; it was a noncomparative study with limited follow-up period of one year. Due to being non-comparative, the refractive status was not evaluated and because of the small sample size, no significant association between BW, GA, PMA, and risk factor could be detected.

In summary, approximately $10 \%$ of ROP cases in the Indian subcontinent is APROP. ${ }^{[4]}$ Need for longterm follow-up and retreatment, concern about systemic complications and financial constraints still loom large in the Indian subcontinent where compliance is a big challenge. Laser therapy can be considered as primary modality of treatment specially in patients with expected poorer compliance and financial constraints.

\section{Financial Support and Sponsorship}

Nil.

\section{Conflicts of Interest}

There are no conflicts of interest.

\section{REFERENCES}

1. Gopal L, Sharma T, Ramachandran S, Shanmugasundaram R, Asha V. Retinopathy of prematurity: a study. Ind J Ophthal 1995;43:59-61.

2. Charan R, Dogra MR, Gupta A, Narang A. The incidence of retinopathy of prematurity in a neonatal care unit. Ind $J$ Ophthalmol 1995;43:123-126.

3. Varughese S, Jain S, Gupta N, Singh S, Tyagi V, Puliyel JM. Magnitude of the problem of retinopathy of prematurity. Experience in a large maternity unit with a medium size level-3 nursery. Ind J Ophthalmol 2001;49:187-188.

4. Hungi B, Vinekar A, Datti N, Kariyappa P, Braganza S, Chinnaiah S, et al. Retinopathy of prematurity in a rural neonatal intensive care unit in South India-a prospective study. Ind J Ped 2012;79:911-915.

5. Nicoară SD, Nascutzy C, Cristian C, Irimescu I, Ștefănuţ AC, Zaharie G, et al. Outcomes and prognostic factors of intravitreal bevacizumab monotherapy in zone I stage $3+$ and aggressive posterior retinopathy of prematurity. $J$ Ophthalmol 2015;2015:102582.

6. Sanghi G, Dogra MR, Katoch D, Gupta A. Aggressive posterior retinopathy of prematurity: risk factors for retinal detachment despite confluent laser photocoagulation. Am J Ophthalmol 2013;155:159-164.e2.

7. Drenser KA, Trese MT, Capone A Jr. Aggressive posterior retinopathy of prematurity. Retina 2010;30:S37-S40.

8. Pandya HK, Faia LJ, Robinson J, Drenser KA. Macular development in aggressive posterior retinopathy of prematurity. Biomed Res Int 2015;2015:808639.

9. Mintz-Hittner HA, Kennedy KA, Chuang AZ, BEAT-ROP Cooperative Group. Efficacy of intravitreal bevacizumab for stage $3+$ retinopathy of prematurity. $N$ Engl J Med 2011;364:603-615.

10. Gunay M, Celik G, Gunay BO, Aktas A, Karatekin G, Ovali F, et al. Evaluation of 2-year outcomes following intravitreal bevacizumab (IVB) for aggressive posterior retinopathy of prematurity. Arq Bras Oftalmol 2015;78:300-304.

11. Nicoară SD, Ştefănuț AC, Nascutzy C, Zaharie GC, Toader LE, Drugan TC, et al. Regression rates following the treatment of aggressive posterior retinopathy of prematurity with bevacizumab versus laser: 8-year retrospective analysis. Med Sci Monit 2016;22:1192-1209.

12. International Committee for the Classification of Retinopathy of Prematurity. The international classification of retinopathy of prematurity revisited. Arch Ophthalmol 2005;123:991-999. 
13. Gunn DJ, Cartwright DW, Gole GA. Prevalence and outcomes of laser treatment of aggressive posterior retinopathy of prematurity. Clin Exp Ophthalmol 2014;42:459-465.

14. Ahn YJ, Hong KE, Yum HR, Lee JH, Kim KS, Youn YA, et al. Characteristic clinical features associated with aggressive posterior retinopathy of prematurity. Eye 2017;31:924-930.

15. Tong $\mathrm{Q}$, Yin $\mathrm{H}$, Zhao $\mathrm{M}$, Li X, Yu W. Outcomes and prognostic factors for aggressive posterior retinopathy of prematurity following initial treatment with intravitreal ranibizumab. BMC Ophthalmol 2018;18:150.

16. Lorenz B, Stieger K, Jäger M, Mais C, Stieger S, AndrassiDarida $\mathrm{M}$, et al. Retinal vascular development with 0.312 mg intravitreal bevacizumab to treat severe posterior retinopathy of prematurity: a longitudinal fluorescein angiographic study. Retina 2017;37:97-111.

17. Mintz-Hittner HA, Geloneck MM, Chuang AZ. Clinical management of recurrent retinopathy of prematurity after intravitreal bevacizumab monotherapy. Ophthalmology 2016;123:1845-1855.

18. Blair M, Garcia Gonzalez JM, Snyder L, Schechet S, Greenwald M, Shapiro M, et al. Bevacizumab or laser for aggressive posterior retinopathy of prematurity. Taiwan $\mathrm{J}$ Ophthalmol 2018;8;243-248.

19. Hu J, Blair MP, Shapiro MJ, Lichtenstein SJ, Galasso JM, Kapur R. Reactivation of retinopathy of prematurity after bevacizumab injection. Arch Ophthalmol 2012;130:10001006.

20. Nicoară SD, Ștefănuț AC, Nascutzy FC, Zaharie GC, Toader LE, Drugan TC. Regression rates following the treatment of aggressive posterior retinopathy of prematurity with bevacizumab versus laser: 8-year retrospective analysis. Med Sci Monit 2016;22:1192-1209.

21. Lepore D, Quinn GE, Molle F, Baldascino A, Orazi L, Sammartino $M$, et al. Intravitreal bevacizumab versus laser treatment in type 1 retinopathy of prematurity: report on fluorescein angiographic findings. Ophthalmology 2014;121:2212-2219.

22. Yonekawa Y, Wu W-C, Nitulescu CE, Paul Chan RV, Thanos A, Thomas BJ, et al. Progressive retinal detachment in infants with retinopathy of prematurity treated with intravitreal bevacizumab or ranibizumab. Retina 2018;38:1079-1083.

23. Carneiro AM, Costa R, Falcão MS, Barthelmes D, Mendonça LS, Fonseca SL, et al. Vascular endothelial growth factor plasma levels before and after treatment of neovascular age-related macular degeneration with bevacizumab or ranibizumab. Acta Ophthalmol 2012;90:e25-e30.

24. Wang D, Choi CS, Lee SJ. Serum concentration of vascular endothelial growth factor after bilateral intravitreal injection of bevacizumab. Korean J Ophthalmol 2014;28:32-38.

25. Sato T, Wada K, Arahori H, Kuno N, Imoto K, IwahashiShima C, et al. Serum concentrations of bevacizumab (Avastin) and vascular endothelial growth factor in infants with retinopathy of prematurity. Am J Ophthalmol 2012;153:327-333.

26. Wu W-C, Lien R, Liao P-J, Wang N-K, Chen Y-P, A Chao, et al. Serum levels of vascular endothelial growth factor and related factors after intravitreous bevacizumab injection for retinopathy of prematurity. JAMA Ophthalmol 2015;133;391-397.

27. Hong YR, Kim YH, Kim SY, Nam GY, Cheon HJ, Lee SJ. Plasma concentrations of vascular endothelial growth factor in retinopathy of prematurity after intravitreal bevacizumab injection. Retina 2015;35:1772-1777.

28. Yang $\mathrm{CH}$. Anti-vascular endothelium growth factor therapy for retinopathy of prematurity: a continuing debate. Taiwan J Ophthalmol 2012;2:115-116.

29. Martínez-Castellanos MA, Schwartz S, Hernández-Rojas ML, Kon-Jara VA, García-Aguirre G, Guerrero-Naranjo JL, et al. Long-term effect of anti-angiogenic therapy for retinopathy of prematurity up to 5 years of follow up. Retina 2013;33:329-338.

30. Morin J, Mai Luu T, Superstein R, Ospina LH, Lefebvre F, Simard M-N, et al. Neurodevelopmental outcomes following bevacizumab injections for retinopathy of prematurity. Pediatrics 2016;137:4.

31. Wu LH, Yang $\mathrm{YH}$, Lin $\mathrm{CH}$, Lin $\mathrm{YJ}$, Cheng $\mathrm{CL}$. Hypotension associated with intravitreal bevacizumab therapy for retinopathy of prematurity. Pediatrics 2016;137:e20152005.

32. Fernandez MP, Berrocal AM, Goff TC, Ghassibi MP, Armitage Harper C, Chou E, et al. Histopathologic characterization of the expression of vascular endothelial growth factor in a case of retinopathy of prematurity treated with ranibizumab. Am J Ophthalmol 2017;176:134140.

33. Moran S, O'Keefe M, Hartnett C, Lanigan B, Murphy J, Donoghue $\mathrm{V}$. Bevacizumab versus diode laser in stage 3 posterior retinopathy of prematurity. Acta Ophthalmol 2014;92:e496-e497.

34. Araz-Ersan B, Kir N, Tuncer S, Aydinoglu-Candan O, Yildiz-Inec D, Akdogan B, et al. Preliminary anatomical and neurodevelopmental outcomes of intravitreal bevacizumab as adjunctive treatment for retinopath of prematurity. Curr Eye Res 2014;15:1-7.

35. Hartnett ME, Penn JS. Mechanisms and management of retinopathy of prematurity. N Engl J Med 2012;367:25152526.

36. Quinn GE, Dobson V, Hardy RJ, Tung B, Palmer EA, Good $\mathrm{WV}$, et al. Visual field extent at 6 years of age in children who had high-risk prethreshold retinopathy of prematurity. Arch Ophthalmol 2011;129:127-132.

37. O'Connor AR, Wilson CM, Fielder AR. Ophthalmological problems associated with preterm birth. Eye 2007;21:1254-1260.

38. Larsson E, Rydberg A, Holmstrom G. Contrast sensitivity in 10 year old preterm and full term children: a population based study. Br J Ophthalmol 2006;90:87-90.

39. Dobson V, Quinn GE, Abramov I, Hardy RJ, Tung $\mathrm{B}$, Siatkowski RM, et al. Color vision measured with pseudoisochromatic plates at five-and-a-half years in eyes of children from the CRYO-ROP study. Invest Ophthalmol Vis Sci 1996;37:2467-2474.

40. Hwang CK, Hubbard GB, Hutchinson AK, Lambert SR. Outcomes after intravitreal bevacizumab versus laser photocoagulation for retinopathy of prematurity: a 5-year retrospective analysis. Ophthalmology 2015;122:10081015.

41. Geloneck MM, Chuang AZ, Clark WL, Hunt MG, Norman AA, Packwood EA, et al. Refractive outcomes following 
bevacizumab monotherapy compared with conventional laser treatment: a randomized clinical trial. JAMA Ophthalmol 2014;132:1327-1333.

42. Kuo HK, Sun IT, Chung MY, Chen YH. Refractive error in patients with retinopathy of prematurity after laser photocoagulation or bevacizumab monotherapy. Ophthalmologica 2015;234:211-217.

43. Gunay M, Celik G, Gunay BO, Aktas A, Karatekin G, Ovali F. Evaluation of 2-year outcomes following intravitreal bevacizumab (IVB) for aggressive posterior retinopathy of prematurity. Arq Bras Oftalmol 2015;78:300-304.

44. Isaac M, Mireskandari K, Tehrani N. Treatment of type 1 retinopathy of prematurity with bevacizumab versus laser. J AAPOS 2015;19:140-144.

45. Tan Q-Q, Christiansen SP, Wang J. Development of refractive error in children treated for retinopathy of prematurity with anti-vascular endothelial growth factor (anti-VEGF) agents: a meta-analysis and systematic review. PLoS One 2019;14:12. 